\title{
Diagnosis and genotyping of African swine fever viruses from 2015 outbreaks in Zambia
}

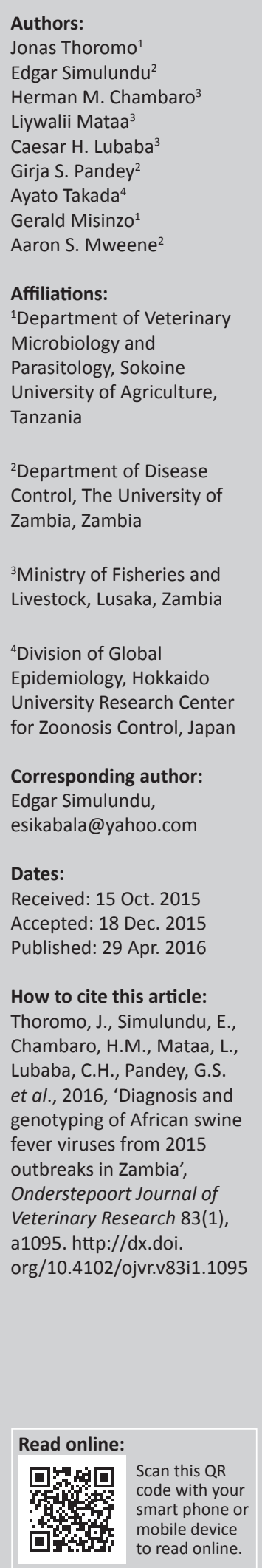

In early 2015, a highly fatal haemorrhagic disease of domestic pigs resembling African swine fever (ASF) occurred in North Western, Copperbelt, and Lusaka provinces of Zambia. Molecular diagnosis by polymerase chain reaction targeting specific amplification of p72 (B646L) gene of ASF virus (ASFV) was conducted. Fourteen out of 16 domestic pigs from the affected provinces were found to be positive for ASFV. Phylogenetic analyses based on part of the p72 and the complete p54 (E183L) genes revealed that all the ASFVs detected belonged to genotypes I and Id, respectively. Additionally, epidemiological data suggest that the same ASFV spread from Lusaka to other provinces possibly through uncontrolled and/or illegal pig movements. Although the origin of the ASFV that caused outbreaks in domestic pigs in Zambia could not be ascertained, it appears likely that the virus may have emerged from within the country or region, probably from a sylvatic cycle. It is recommended that surveillance of ASF, strict biosecurity, and quarantine measures be imposed in order to prevent further spread and emergence of new ASF outbreaks in Zambia.

\section{Introduction}

African swine fever (ASF) is a highly contagious and fatal haemorrhagic disease of domestic pigs caused by a complex DNA virus of the genus Asfivirus, family Asfarviridae. Most isolates of ASF virus (ASFV) cause an acute haemorrhagic fever with mortality rates that can reach $100 \%$ in susceptible pigs. ASFV circulates in an ancient sylvatic cycle involving soft ticks of the Ornithodoros species and warthogs (Phacochoerus aethiopicus) as well as in domestic pig populations with or without involvement of Ornithodoros ticks; other wild suids, in particular bushpigs (Potamochoerus larvatus, Potamochoerus porcus) can act as asymptomatic reservoirs of the virus but are not associated with ticks and their role, if any, in the epidemiology of the disease is unknown (Muhangi 2014).

Although ASF is known to be endemic in sub-Saharan Africa (Penrith et al. 2013), it is occasionally introduced into new regions or continents. For example, in June 2007, ASF was introduced into the Republic of Georgia and subsequently spread into neighbouring countries including Armenia, Azerbajan, the Russian Federation, Ukraine, and Belarus (Beltrán-Alcrudo et al. 2008; Chapman et al. 2011). Recently, ASF has spread into some of the member states of the European Union including Poland, Latvia, Lithuania, and Estonia (Pejsak et al. 2014). These events stress the need for concerted international efforts in the control of ASF.

In Zambia, ASF was first reported in 1912 in the vicinity of Fort Jameson, now called Chipata, in Eastern Province (Wilkinson et al. 1988). Subsequently, there have been repeated reports of its occurrence in the same area, where it is now considered to be endemic. Laboratory confirmation was done on only five occasions between 1974 and 1983 (Wilkinson et al. 1988). However, a recent study confirmed an outbreak of ASF in Lusaka in October 2013 (Yabe et al. 2015). Although the Zambian government has endeavoured to control the disease, outbreaks continue to be reported across the country in non-endemic regions (Yabe et al. 2015). In early 2015, outbreaks of a haemorrhagic disease resembling ASF that killed a number of domestic pigs were reported in North Western, Copperbelt, and Lusaka provinces of Zambia. The present study was aimed at confirming and conducting genotypic characterisation of ASFVs involved in these outbreaks.

\section{Materials and methods}

\section{Study area, sample collection, and processing}

Tissue samples were collected from domestic pigs in districts with suspected ASF cases based on clinical signs, post-mortem lesions, and reports from local veterinary officials and farmers during January to April 2015. Samples were obtained from Kitwe district in the Copperbelt province; Chongwe, Chilanga (Linda area), and Lusaka (Chamba valley area) in Lusaka province; and

Copyright: @ 2016. The Authors. Licensee: AOSIS. This work is licensed under the Creative Commons Attribution License. 
Solwezi district in North Western province (Figure 1). Eight samples were collected from domestic pigs in Lusaka province, four samples came from North Western, and four from the Copperbelt provinces, making a total of 16 . Tissues collected included mesenteric lymph nodes, spleen, kidney, and liver. Samples were collected in sterile tubes and transported on ice to the laboratory where they were stored at $-80{ }^{\circ} \mathrm{C}$ until use. Tissues were homogenised in phosphatebuffered saline at a ratio of 1:10 (w/v). Tissue homogenates from each animal were pooled and used for laboratory diagnosis and molecular analyses.

\section{DNA extraction}

One $\mu \mathrm{L}$ of Proteinase K $(20 \mathrm{mg} / \mathrm{mL})$ was added to $100 \mu \mathrm{L}$ of $10 \%$ tissue homogenate followed by overnight incubation of the tissue-enzyme mixture at $55^{\circ} \mathrm{C}$. Then the tissue-enzyme mixture was heated at $95{ }^{\circ} \mathrm{C}$ for $10 \mathrm{~min}$ followed by centrifugation at $6000 \mathrm{~g}$ for $5 \mathrm{~min}$. The supernatant containing DNA was collected and the pellet discarded.

\section{Diagnosis of African swine fever virus using polymerase chain reaction}

ASFV DNA was detected by polymerase chain reaction (PCR) using PPA1/2 primers, as previously reported by Agüero et al. (2003). PCR was carried out in Gene Amp PCR System (Applied Bio-systems, Foster City, CA USA) using Taq polymerase (Thermoscientific, Waltham, MA USA), according to the manufacturer's instructions. PCR products were separated by electrophoresis on a 1.5\% agarose gel stained with GelRed nucleic acid stain (Phenix Research Products, Candler, USA).

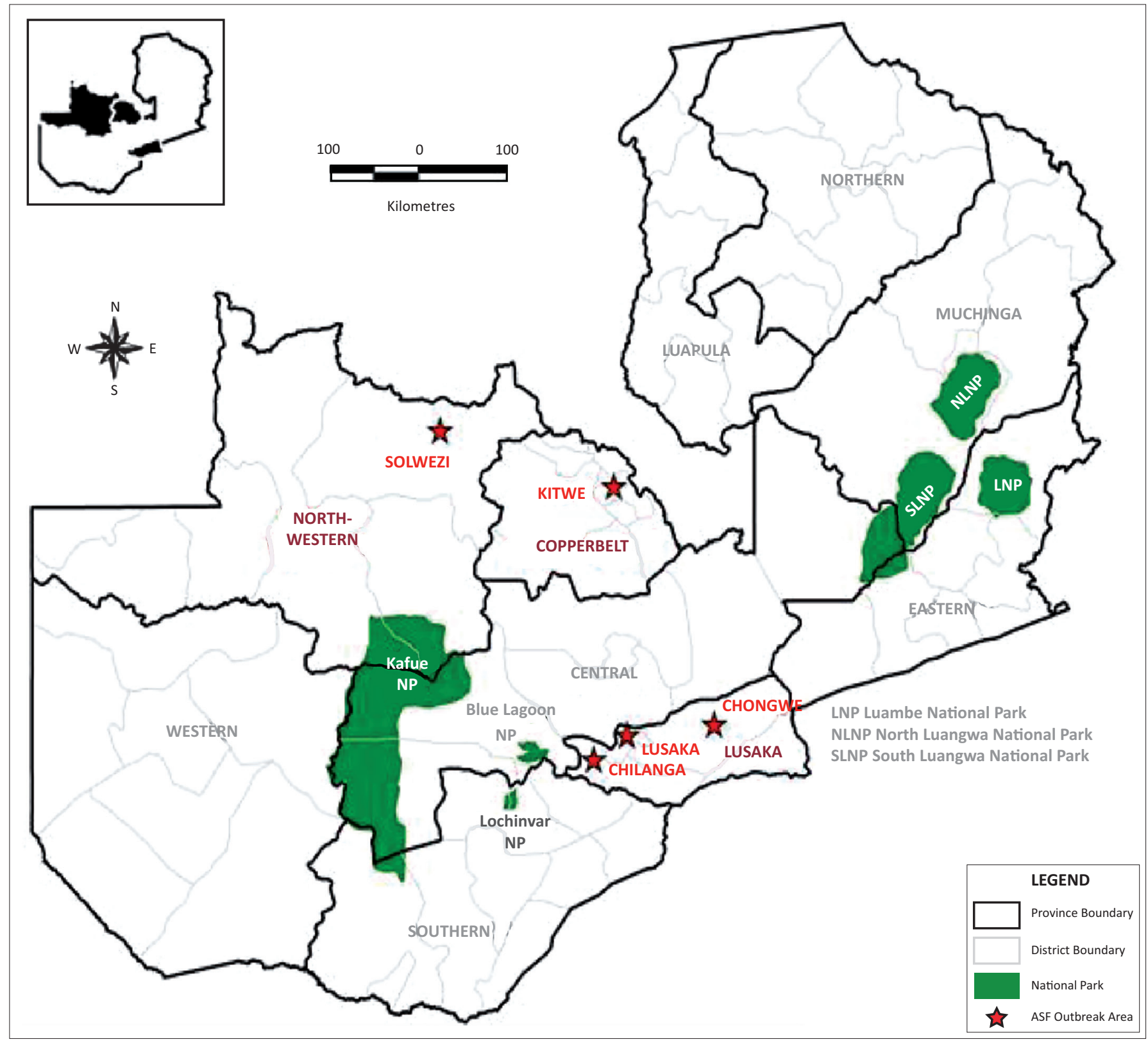

Source: Authors own creation (generated by Arc View software)

Note: The areas where the outbreak occurred are indicated by stars.

FIGURE 1: Map showing sampling locations for African swine fever in domestic pigs. 


\section{Genotyping of African swine fever virus}

Genotyping of ASFV was performed by partial amplification of the p72 (B646L) and complete p54 (E183L) gene amplification using PCR. Primers including P72U/D and PPA89/722 were used in the amplification of p72 and p54 genes, respectively (Bastos et al. 2003; Nix et al. 2006). The amplification conditions previously described by Gallardo et al. (2009) were used in the present study. PCR products were purified from agarose gels using a NucleoSpin gel and PCR clean-up kit (Macherey-Nagel, Düren, Germany) and subjected to dideoxynucleotide cycle sequencing using Big Dye Terminator Cycle Sequencing Kit Version 3.1 (Applied Biosystems, Foster City, CA USA). Products from dideoxynucleotide cycle sequencing reaction were purified by ethanol precipitation and separated on a 3500 Genetic Analyzer (Applied Biosystems, Foster City, CA USA). Chromatograms for both the forward and the reverse primer reactions were read using Sequence Scanner v1.0 software (Applied Biosystems, Foster City, CA USA). The obtained sequences were deposited in GenBank/EMBL/ DDBJ databases under accession numbers LC088171LC088176.

\section{Data analysis}

ASFV nucleotide sequences representing the 22 p72 genotypes (Boshoff et al. 2007) were obtained from GenBank database and analysed in MEGA 6.06 (Tamura et al. 2013) along with those determined in the present study. The $\mathrm{p} 72$ and p54 nucleotide sequences were aligned in MEGA 6.06 using Clustal W. Phylogenetic reconstruction was conducted using the neighbour-joining method. Phylogenetic tree reliability was supported using the bootstrap method with 1000 replications.

\section{Results \\ Outbreak history}

In Solwezi district in North western province, an outbreak of a pig disease similar to ASF was observed in JanuaryFebruary 2015 at a farm with a total of 66 pigs. Twenty pigs died as a result of the disease whereas 46 were depopulated by government. At another farm in the same district 7 pigs died from a similar disease and the rest $(23$ pigs) were depopulated by government. Epidemiological investigations revealed that all the pigs at the primary farm were brought from the Copperbelt province. During this time period, 4 pigs died from a herd of 68 at a farm in Kitwe district of the Copperbelt. The rest of the pigs (64) were depopulated by government officials. The origin or source of the disease was not established. In Chongwe district (Lusaka province), the disease came about after a boar was bought from Kaunda square in Lusaka from a farmer whose pigs had started dying and was selling them off. The farmer in question had initially bought the pigs from Chilanga district. This was during a time when it was not mandatory to test animals meant for breeding for ASF. The case was reported in April 2015. During the sampling period, more than 600 pigs died in Lusaka province including Chilanga (Linda area), Chongwe, and Lusaka (Chamba valley area) either through depopulation or from disease.

\section{Clinical signs and post-mortem findings}

Clinical signs and gross pathological findings of the screened animals in this study were similar to those of ASF, and hence this disease was highly suspected. The animals presented with incoordination, inappetence, cyanosis of the ears, petechial haemorrhages on leg extremities, tachypnoea, and recumbence. On post-mortem examination pigs had oedematous lungs, fluids in body cavities, and haemorrhages and congestion in internal organs such as the spleen, lymph nodes, intestine, and kidneys.

\section{Detection of African swine fever virus genome}

In total, 16 clinical samples from four different districts in Zambia were analysed using PCR. Out of the 16 samples, ASFV was detected in 14 samples using three different pairs of primers that specifically amplify different parts of ASFV genome including $B 646 \mathrm{~L}$ and $E 183 \mathrm{~L}$.

\section{Nucleotide sequence and phylogenetic analysis}

The p72 and p54 nucleotide sequences of ASFV detected in domestic pigs in Kitwe, Solwezi, Chongwe, Lusaka, and Chilanga were found to be $100 \%$ similar to each other.

Phylogenetic analysis of the p72 gene sequences from this study clustered into genotype I (Figure 2a). Similarly, phylogenetic analysis of the E183L (p54) nucleotide sequences showed that the 2015 Zambian ASFV belonged to genotype Id (Figure 2b). Moreover, they were different from other genotype I ASFVs such as those from West Africa (genotype Ib) and America and Europe (genotype Ia and Ic) (Figure 2b).

\section{Discussion}

Although Zambia has experienced many outbreaks of ASF, genetic characterisation of ASFVs involved in outbreaks has been conducted only for some viruses. Available genotyping studies were performed before the year 2008 (Lubisi et al. 2005, 2007; Nix et al. 2006). However, ASF outbreaks in domestic pigs have continued to occur in Zambia, including the one reported in 2013 (Yabe et al. 2015) and those reported in this study.

In this study, clinical and post-mortem findings consistent with those of ASF were observed suggesting that outbreaks of a highly fatal haemorrhagic disease afflicting domestic pigs in North Western, Copperbelt, and Lusaka provinces of Zambia could be ASF. ASFV infection of diseased pigs was confirmed through detection of different parts of the ASFV genome by PCR. The finding that nucleotide sequences of the p72 and p54 genes of ASFV under study were 100\% identical suggests that these outbreaks were caused by the same virus. 

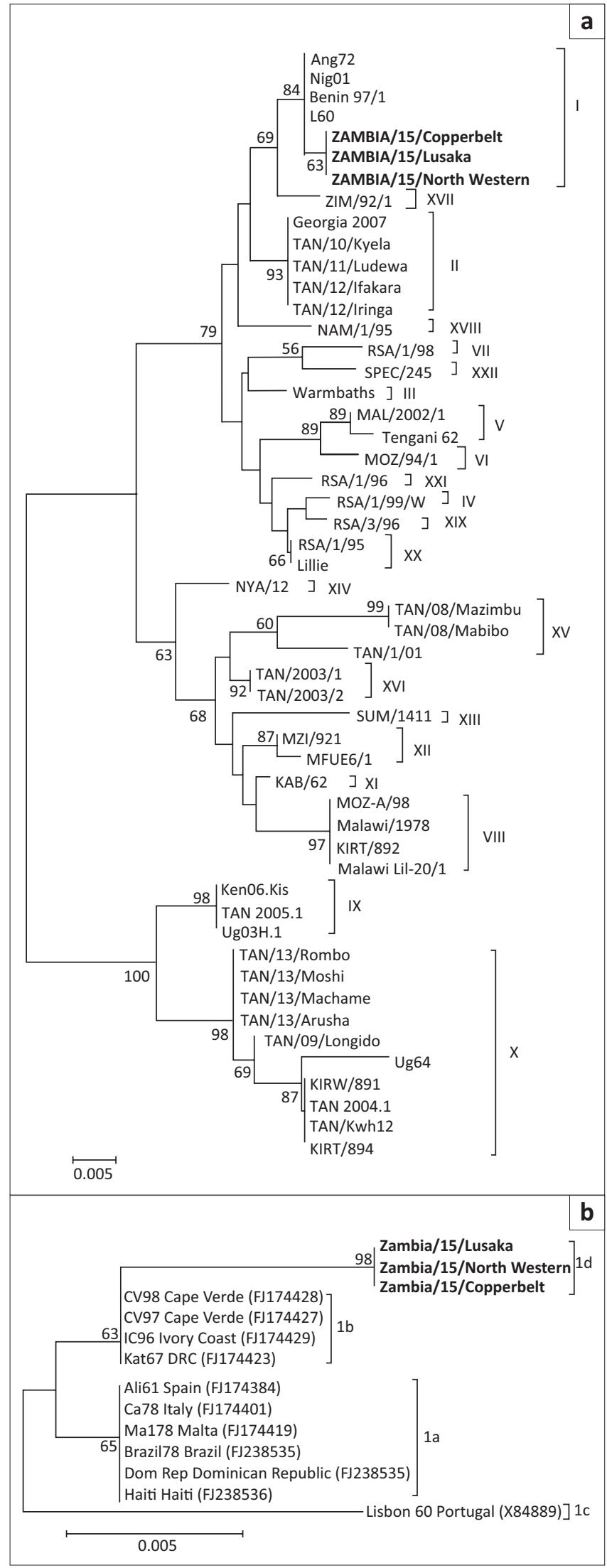

Source: Authors own creation (generated by MEGA 6 software)

Note: Genotypes are denoted by Roman numerals adjacent to square brackets. Numbers at branch nodes indicate neighbour-joining bootstrap values of $\geq 50 \%$. ASFVs characterised in this study are in bold. Bars, number of substitutions per site.

FIGURE 2: Phylogenetic relationships based on nucleotide sequences of part of p72 (a) and p54 (b) genes of African swine fever virus detected in tissues collected from diseased pigs in Zambia.
Phylogenetic analyses based on the p72 nucleotide sequences confirmed that the 2015 ASFV from Zambia belonged to genotype I (Figure 2a). Genotype I ASFVs have a wide geographical distribution and have been previously reported in Europe, South America, the Caribbean Islands, West Africa, East Africa, and southern Africa (Lubisi et al. 2005). Based on the finding that the p54 nucleotide sequences of 2015 Zambian ASFV belonged to the southern African genotype $1 \mathrm{~d}$, it seems likely that this virus may have originated from within the country (or possibly from the southern African region) and may not have been imported from other geographical locations.

Epidemiological data gathered from farmers and veterinarians among the different provinces of Zambia during the 2015 ASF outbreaks suggest that ASF spread within Zambia was facilitated by movement of infected pigs and/or pig products from affected areas to disease free areas. For instance, in Chongwe district (Lusaka province), the disease came about after a boar was bought from Kaunda square (Lusaka district) from a farmer who was selling off pigs because his pigs had started dying. Similarly, ASF broke out in Solwezi after a farmer purchased pigs from the Copperbelt province. It is therefore recommended that future control and prevention strategies of ASF in Zambia should focus on early detection through screening of the animals and timely confirmation of the disease, strict quarantine measures, biosecurity, stock movement restrictions including culling and proper disposal of infected and in-contact animals, and decontamination of affected premises.

\section{Acknowledgements}

We thank participating farmers and veterinarians in Zambia. This work was supported in part by the Japan Initiative for Global Research Network on Infectious Diseases and Japan Science and Technology Agency/Japan International Cooperation Agency within the framework of the Science and Technology Research Partnership for Sustainable Development. J.T. was supported by a scholarship from the Wellcome Trust (Grant WT087546MA) to Southern African Centre for Infectious Diseases Surveillance, Sokoine University of Agriculture, Morogoro, Tanzania.

\section{Competing interests}

The authors declare that they have no financial or personal relationships which may have inappropriately influenced them in writing this article.

\section{Authors' contributions}

J.T. and E.S. conducted most of the experiments. E.S. and G.M. were responsible for experimental and project design. H.M.C. and G.S.P. participated in sample collection and preparation. L.M. and C.H.L. were involved in epidemiological investigations and data analysis. A.T., G.M. and A.S.M. were project leaders and made conceptual contributions. All authors participated in writing of the manuscript. 


\section{References}

Agüero, M., Fernández, J., Romero, L., Sánchez Mascaraque, C., Arias, M. \& SánchezVizcaíno, J.M., 2003, 'Highly sensitive PCR assay for routine diagnosis of African swin fever virus in clinical samples', Journal of Clinical Microbiology 41(9), 4431-4434.

Bastos, A.D.S., Penrith, M.L., Crucière, C., Edrich, J.L., Hutchings, G., Roger, F. et al., 2003, 'Genotyping field strains of African swine fever virus by partial p72 gene characterization', Archives of Virology 148(4), 693-706.

Beltrán-Alcrudo, D., Lubroth, J., Depner, K. \& De La Rocque, S., 2008, 'African swine fever in the Caucasus', Empres Watch (April), 1-8.

Boshoff, C.I., Bastos, A.D., Gerber, L.J. \& Vosloo, W., 2007, 'Genetic characterisation of African swine fever viruses from outbreaks in southern Africa', Veterinary Microbiology 121(2), 45-55.

Chapman, D.A.G., Darby, A.C., Da Silva, M., Upton, C., Radford, A.D. \& Dixon, L.K., 2011, 'Genomic analysis of highly virulent Georgia 2007/1 isolate of African swine fever virus', Emerging Infectious Diseases 17(4), 599-605.

Gallardo, C., Mwaengo, D.M., Macharia, J.M., Arias, M., Taracha, E.A., Soler, A. et al., 2009, 'Enhanced discrimination of African swine fever virus isolates through nucleotide sequencing of the p54, p72, and pB602L (CVR) genes', Virus Genes 38(1), 85-95.

Lubisi, B.A., Bastos, A.D., Dwarka, R.M. \& Vosloo, W., 2005, 'Molecular epidemiology of African swine fever in East Africa', Archives of Virology 150, 2439-2452.
Lubisi, B.A., Bastos, A.D., Dwarka, R.M. \& Vosloo, W., 2007, 'Intra-genotypic resolution of African swine fever viruses from an East African domestic pig cycle: A combined p72-CVR approach', Virus Genes 35(3), 729-735.

Muhangi, D., 2014, 'African swine fever: An epidemiological overview', British Journal of Virology 1, 42-47.

Nix, R.J., Gallardo, C., Hutchings, G., Blanco, E. \& Dixon, L.K., 2006, 'Molecular epidemiology of African swine fever virus studied by analysis of four variable genome regions', Archives of Virology 151, 2475-2494.

Pejsak, Z., Truszczyński, M., Niemczuk, K., Kozak, E. \& Markowska-Daniel, I., 2014, 'Epidemiology of African swine fever in Poland since the detection of the first case', Poland Journal of Veterinary Science 17(4), 665-672.

Penrith, M.L., Vosloo, W., Jori, F. \& Bastos, A.D.S., 2013, 'African swine fever virus eradication in Africa', Virus Research 173(1), 228-246.

Tamura, K., Stecher, G., Peterson, D., Filipski, A. \& Kumar, S., 2013, 'MEGA6: Molecular Evolutionary Genetics Analysis version 6.0', Molecular Biology and Evolution 30 2725-2729.

Wilkinson, P.J., Pegram, R.G., Perry, B.D., Lemche, J. \& Schels, H.F., 1988, 'The distribution of African swine fever virus isolated from Ornithodoros moubata in Zambia', Epidemiology and Infection 101(3), 547-564.

Yabe, J., Hamambulu, P., Simulundu, E., Ogawa, H., Kajihara, M., Mori-Kajihara, A. et al., 2015, 'Pathological and molecular diagnosis of the 2013 African swine fever outbreak in Lusaka, Zambia', Tropical Animal Health and Production 47(2), 459-463. 